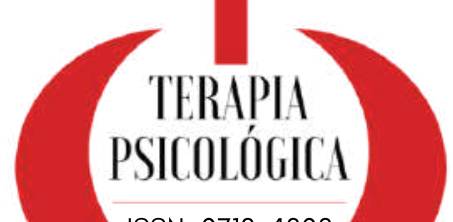

ISSN: 0718-4808

\title{
Problemas de regulación emocional y salud mental en adolescentes del norte de Chile
}

\author{
Emotional regulation problems and mental health in \\ adolescents in northern Chile
}

\author{
Alejandra Caqueo-Urízar \\ (iD) 0000-0002-4614-8380 \\ acaqueo@academicos.uta.cl \\ Universidad de Tarapacá. \\ Arica, Chile.
}

\section{Marigen Narea (iD) 0000-0001-7780-7425 mnarea@uc.cl \\ Pontificia Universidad Católica de Chile. Santiago, Chile. \\ Centro de Justicia Educacional.}

\section{Resumen:} comportamientos disruptivos.

\section{Abstract:}

\author{
Patricio Mena-Chamorro \\ (iD) 0000-0001-7397-2845 \\ pmenaeuta.cl \\ Universidad de Tarapacá . \\ Arica, Chile. \\ Centro de Justicia Educacional.
}

\author{
Jerome Flores \\ (iD) 0000-0001-9333-6870 \\ jflores@uta.cl \\ Universidad de Tarapacá . \\ Arica, Chile. \\ Centro de Justicia Educacional.
}

Matías Irarrázabal

(iD) 0000-0002-9037-8305

mirarrazabaldeu.uchile.cl

Universidad de Chile, Santiago, Chile.

El propósito de este estudio fue evaluar la asociación entre los problemas de regulación emocional e indicadores de salud mental en adolescentes de la ciudad Arica al Norte de Chile, además de describir y comparar los niveles de problemas interiorizados y exteriorizados entre hombres y mujeres. Participaron 2331 estudiantes de 11 a 20 años provenientes de establecimientos educacionales. Para medir los problemas de regulación emocional y los indicadores de salud mental, se utilizó el Sistema de Evaluación de Niños y Adolescentes (SENA). Los resultados muestran que los problemas de regulación emocional poseen efectos grandes y significativos sobre los problemas interiorizados y exteriorizados. Asimismo, se observó que las mujeres presentan mayor sintomatología depresiva, ansiosa, de ansiedad social y problemas de regulación emocional que los hombres. Pareciera ser que los adolescentes que manifiestan problemas para regular sus emociones son más vulnerables a experimentar estados de ánimos disfóricos, preocupaciones recurrentes o

Palabras Clave: Regulación emocional, Salud Mental, Adolescentes, SENA

The aim of this study was to assess the relationship between emotional regulation problems and mental health outcomes in adolescents from the city of Arica in northern Chile, in addition to describing and comparing the levels of internalized and externalized problems between men and women. A total of 2331 students from 11 to 20 years old from educational establishments participated in the study. To measure emotional regulation problems and mental health indicators, the System of Evaluation of Children and Adolescents (SENA) was used. The results show that emotional regulation problems have large and significant effects on both internalized and externalized problems. Likewise, it was observed that women show more depressive, anxious, social anxiety and emotional regulation problems than men. It seems that adolescents who manifest problems in regulating their emotions are more vulnerable to experience dysphoric moods, recurrent worries or disruptive behavior.

Keywords: Emotional regulation, Mental Health, Adolescents, SENA 


\section{Introducción}

La regulación emocional se puede definir como un conjunto de procesos intrínsecos y extrínsecos que, en base a la activación de un objetivo, controlarán el inicio, mantención, y modificación de reacciones emocionales positivas y negativas (Gross y Thompson, 2007; Thompson, 1994), a través de diversos procesos cognitivos que, además influirán en la latencia, intensidad, y duración de dicha emoción (Gross, Sheppes, Urry, 2011). Este impacto en la dinámica de las emociones permite que los sujetos formen sus emociones, aludiendo al cómo y cuándo las experimentan (Barrett, Ochsner y Gross, 2007).

Durante las dos últimas décadas, la investigación sobre regulación emocional ha aumentado significativamente, siendo una de las áreas más fructíferas y de rápido crecimiento (Tamir, 2011). Los diversos campos de investigación convergen en que las dificultades para regular las emociones poseen un rol fundamental en el desarrollo y mantenimiento de sintomatología psicopatológica, sobre todo, en población infanto-juvenil (Aldao, Gee, De Los Reyes y Seager, 2016; Gratz, Weiss y Tull, 2015; Berking, Wirtz, Svald y Hofmann, 2014, Lee et al., 2014; Aldao y Nolen-Hoeksema, 2010; Eisenberg, Spinrad y Eggum, 2010).

Los constantes cambios en el funcionamiento biológico, cognitivo, social y familiar característicos de la adolescencia (Blakemore y Mills, 2014), exponen a los adolescentes a mayores fuentes de estrés y emociones intensas, como la separación de los padres, bullying, rendimiento académico, interacción con sus compañeros, entre muchas otras (Cracco, Goossens y Braet, 2017; de Veld, Riksen-Walraven y de Weerth, 2012). Convirtiendo a los jóvenes en personas más vulnerables a influencias internas y sociales (Bailen, Green y Thompson, 2019; Ahmed, Bittencourt-Hewitt y Sebastian, 2015).

La alta prevalencia de trastornos mentales presentados en la adolescencia (Polanczyk, Salum, Sugaya, Caye y Rohde, 2015) y la posibilidad de que las experiencias de los individuos durante este período pueden perpetuarse en la adultez, influyendo y configurando en gran medida sus futuras interacciones sociales y bienestar, es que los investigadores han centrado sus esfuerzos en asociar los problemas de regulación emocional con múltiples factores psicosociales en diversos contextos, destacándose los estudios en el área clínica (Schäfer, Naumann, Holmes, Tuschen-Caffier y Samson, 2017; Oram, Ryan, Rogers y Heath, 2017; Criss, Morris, Ponce Garcia, Cui y Silk, 2016; Dvorak, Sargent, Kilwein, Stevenson, Kuvaas y Williams, 2014; Veilleux, Skinner, Reese y Shaver, 2014). Consecuencia de esto, es la propuesta de la 
Alejandra Caqueo-Urízar, Patricio Mena-Chamorro, Jerome Flores-Jara, Marigen Narea-Biscupovich, Matías Irarrázaval-Domínguez

regulación emocional como un elemento transdiagnóstico en psicoterapia (Sloan, Hall, Moulding, Bryce, Mildred y Staiger, 2017), transformándose en un objetivo central para el tratamiento de: (1) problemas interiorizados, entendidos como dificultades principalmente emocionales que afectan el estado ánimo y preocupación de los adolescentes y (2) problemas exteriorizados, que hacen referencia a comportamientos disruptivos, que en algunas ocasiones suelen ser visualizados por un tercero (Aldao et al., 2016; Braet et al., 2014).

Los hallazgos reportados por estudios en muestras clínicas y no clínicas convergen en que los procesos de regulación emocional adecuados obtienen reducciones significativas en sintomatología ansiógena y depresiva, así como en comportamientos disruptivos (Mennin, Fresco, O'Toole y Heimberg, 2018; Lenz, Del Conte, Hollenbaugh y Callendar, 2016; Garnefski y Kraaij, 2016; Eastabrook, Flynn y Hollenstein, 2013; McLaughlin, Hatzenbuehler, Mennin y NolenHoeksema, 2011; Saxena, Dubey y Pandey, 2011). Estos resultados también se sostienen entre distintas intervenciones terapéuticas que tienen como objetivo el entrenamiento de habilidades específicas de regulación emocional (Ehret, Kowalsky, Rief, Hiller y Berking, 2014; Berking et al., 2008), dentro de las que destacan la terapia de regulación emocional (Mennin y Fresco, 2009), entrenamiento en regulación emocional (Berking, 2010), entrenamiento en procesamiento emocional óptimo (Hervás y Vázquez, 2006).

Si bien, los estudios se han enfocado principalmente en evaluar los efectos de la regulación emocional disfuncional sobre los problemas conductuales y emocionales, también existen estudios que se han orientado a evaluar el rol de las diferencias de género en dicha asociación. Nolan-Hoeksema y Aldao (2011) encontraron que las mujeres eran más propensas a utilizar varias y diferentes estrategias de regulación emocional que los hombres y, que el uso de estrategias de regulación disminuye con la edad, con la excepción de la estrategia supresión que aumentó en mujeres. Así, otros estudios señalan que los hombres se caracterizan por manifestar mayores conductas agresivas, problemas de atención, mientras que en las mujeres se observa mayor sintomatología depresiva y ansiosa (Lee y Bukowski, 2012; Nolen-Hoeksema, 2012). Estas diferencias se pueden atribuir a que los hombres y mujeres expresan sus emociones de manera diferente, utilizando distintas estrategias de regulación emocional (Chaplin, 2015; Chaplin y Aldao, 2013).

A pesar de existir bastante evidencia que fundamenta la asociación entre estas variables, la mayoría de las investigaciones se han realizado en países desarrollados (Estados Unidos y Europa) donde la asignación de recursos a la salud mental es mayor a la que pueden optar 
aquellos países en vías de desarrollo como Chile y el resto de países Latinoamericanos (Saxena, Thornicroft, Knapp y Whiteford, 2007). El gasto público en salud mental en estas regiones, es muy inferior al necesario, sobre todo si se considera la alta prevalencia de trastornos mentales y la baja disponibilidad de intervenciones asequibles para los ciudadanos (Saraceno et al., 2007).

Aunque Chile se caracteriza por entregar una atención de salud mental institucionalizada que permite un mayor acceso en comparación a otros países de Latinoamérica, la escasez de recursos aún persiste (Errázuriz, Valdés, Vöhringer y Calvo, 2015). Por tanto, la necesidad de obtener una mayor comprensión de este fenómeno en países con acceso limitado al tratamiento de salud mental es imprescindible, sobre todo por sus posibles repercusiones en la salud mental de los adolescentes y su esencial contribución en psicoterapia.

El propósito de este estudio es evaluar la asociación entre los problemas de regulación emocional e indicadores de salud mental en adolescentes de la ciudad Arica al Norte de Chile, además de describir y comparar los niveles de problemas interiorizados y exteriorizados entre hombres y mujeres.

\section{Método}

Estudio no experimental cuyo diseño es transversal ex post facto retrospectivo, con alcance correlacional, debido a que se miden todas las variables en un solo momento y el fenómeno ya ha ocurrido (Ato, López-García y Benavente, 2013).

Esta investigación forma parte de un proyecto mayor, llevado a cabo por la Línea de Inclusión Psicosocial del Centro de Justicia Educacional (CJE).

\section{Participantes}

La muestra estuvo constituida por 2331 estudiantes de 11 a 20 años (séptimo básico a cuarto medio) provenientes de establecimientos educacionales de la ciudad de Arica, donde el 45.8\% ( $N=1069$ ), pertenecía a Establecimientos Particular Subvencionado por el Gobierno, $41.8 \% \quad(\mathrm{~N}=973)$ a Municipales y $12.4 \% \quad(\mathrm{~N}=289)$ a escuelas Privadas. La elección de los participantes se realizó a través de una estrategia de muestreo no probabilística, por conveniencia (Otzen y Manterola, 2017). 
En general, la edad media fue de 14.3 (DE = 1.8) años, 1171 (50.5\%) fueron mujeres y 1150 (49.5\%) fueron hombres. Respecto a la nacionalidad de los participantes 2165 (93.3\%) eran chilenos y $156(6.7 \%)$ extranjeros. Los estudiantes presentaron las siguientes puntuaciones medias y desviaciones típicas en: problemas de regulación emocional $(M=2.4 ; D E=1.0)$, depresión ( $M=2.1 ; D E=0.8)$, ansiedad $(M=2.5 ; D E=0.8)$, ansiedad social $(M=2.4 ; D E=0.8)$, problemas de atención $(M=2.3 ; \mathrm{DE}=0.7)$, hiperactividad-impulsividad $(M=2.0 ; \mathrm{DE}=0.6)$ y conducta desafiante $(M=1.8 ; D E=0.7)$, dando cuenta que la mayoría de las respuestas se centraron en reportar una baja frecuencia de problemas para comprender y expresar sus emociones, así como bajos problemas interiorizados y exteriorizados.

\section{Instrumentos}

Escala sociodemográfica ad-hoc: Utilizada para la identificación del sexo, edad y nacionalidad de los estudiantes.

Sistema de evaluación de niños y adolescentes (SENA) (Fernández-Pinto, Santamaría, Sánchez-Sánchez, Carrasco, y del Barrio, 2015a; 2015b): está compuesto por 9 cuestionarios, con el fin de evaluar un amplio espectro de problemas emocionales y conductuales a través de tres grupos de edad: Infantil (3-6 años), Primaria (6-12 años) y Secundaria (12-18 años). Las opciones de respuesta para cada versión, corresponden a declaraciones conductuales en formato Likert de 5 puntos ( 1 = "Nunca" a 5 = "Siempre"). Esta escala reportó evidencia de validez y fiabilidad adecuados para ser utilizado en niños y adolescentes de contextos hispanohablantes (Sánchez-Sánchez, Fernández-Pinto, Santamaría, Carrasco y del Barrio, 2016).

El conjunto de cuestionarios posee un enfoque multidimensional y se encuentra distribuido mediante tres bloques de escalas principales (escalas de problemas, de vulnerabilidad y de recursos personales), que incluyen variables que van desde la depresión (DEP), ansiedad (ANS), problemas de atención (ATE) a búsqueda de sensaciones (BUS), consumo de sustancias (SUS) y problemas con los compañeros (COM). Para el desarrollo de esta investigación se utilizaron los siguientes cuestionarios en su versión Secundaria (12-18 años):

1. Problemas de regulación emocional (REG): evalúa la presencia de dificultades para comprender, regular y expresar las propias emociones, a través de ítems como "Me cuesta entender mis sentimientos". Puntajes altos sugieren que los estudiantes tienen dificultades para controlar y regular sus emociones, así como problemas para identificar y reconocer las 
causas que les hacen experimentar determinadas emociones.

2. Depresión (DEP): mide la presencia de sintomatología depresiva, que se caracteriza por ánimo disfórico, anhedonia, sentimientos de inutilidad, culpa y pensamientos asociados con la muerte. Esta escala contiene ítems como "Sufro mucho". Puntajes altos sugieren la presencia de manifestaciones depresivas, expresadas mediante un estado de ánimo triste o irritable.

3. Ansiedad (ANS): evalúa la presencia de un malestar subjetivo generalizado caracterizado por preocupaciones persistentes y recurrentes propias de la ansiedad generalizada mediante ítems como "Me angustian o agobian mis problemas". Puntajes altos sugieren que los estudiantes manifiestan sensaciones de nerviosismo, malestar subjetivo general y sobreactivación fisiológica.

4. Ansiedad social (ASC): mide la presencia de síntomas ansiosos específicamente asociados con situaciones de tipo social, donde las personas temen a ser evaluadas o enjuiciadas. Esta escala contiene ítems como "Me pongo nervioso cuando hay mucha gente alrededor". Puntajes altos señalan que los estudiantes manifiestan incomodidad, nerviosismo e inseguridad en escenarios sociales.

5. Problemas de atención (ATE): evalúa la presencia de síntomas de inatención característicos del trastorno por déficit de atención e hiperactividad (TDAH), concentrándose en el déficit de control atencional, como dirigir atención hacia la tarea o estímulos irrelevantes. Esta escala contiene ítems como "Me despisto y cometo muchos errores sin darme cuenta". Puntajes altos sugieren que los estudiantes tienen dificultades para mantener y dirigir su atención.

6. Hiperactividad-impulsividad (HIP): mide los comportamientos hiperactivos e impulsivos propios del trastorno por déficit de atención e hiperactividad (TDAH), enfocándose en actividad motriz excesiva y exagerada, además de un déficit en el control inhibitorio. Esta escala contiene ítems como "Me llaman la atención porque corro o salto donde no debo". Puntajes altos sugieren que los estudiantes poseen un nivel motriz excesivo y tienen dificultades para inhibir su conducta.

7. Conducta desafiante (DES): evalúa la presencia de comportamiento de oposición a las figuras de autoridad, como los padres, profesores y otros adultos. Incluye comportamientos de desobediencia en el hogar y clases, hasta conductas hostiles y de enfrentamiento 
problemáticos. Esta escala contiene ítems como "Si mis padres me prohíben algo, lo hago más todavía". Puntajes altos sugieren que los estudiantes ejecutan comportamientos desafiante y desobediente con sus padres, profesores y otros adultos.

\section{Procedimiento}

Se contactó a los Directores y Orientadores de los establecimientos educacionales de Arica, invitándolos a participar de manera voluntaria en este estudio. El proceso de aplicación del instrumento se llevó a cabo a través de papel y lápiz en forma grupal al interior de la clase. La extensión de la evaluación fue aproximadamente 45 minutos y no hubo ninguna gratificación por responder el cuestionario.

\section{Consideraciones éticas}

La presente investigación, fue aprobada por el Comité Ético Científico de la Universidad de Tarapacá.

Antes de iniciar el proceso de aplicación, en las reuniones de apoderados y salas de clases se explicó los objetivos del estudio y se entregó un consentimiento informado a los padres y posteriormente se obtuvo el asentimiento informado de los adolescentes, el cual contenía por escrito el propósito del estudio, las garantías de anonimato y confidencialidad, los derechos de los participantes, los posibles riesgos y los datos de contacto del investigador principal.

\section{Análisis estadístico}

Previo a la estimación del modelo de ecuación estructural y con la finalidad de proporcionar evidencia de validez y fiabilidad preliminar, se procedió a probar y depurar los modelos de medida, mediante análisis factoriales confirmatorios (AFC) y coeficientes alfa de Cronbach y omega jerárquico de McDonald's. Posteriormente, con los modelos de medida depurados, se estimó un modelo de ecuación estructural (SEM), con los efectos de los problemas de regulación emocional (REG) sobre los problemas interiorizados, compuesto por depresión (DEP), ansiedad (ANS), ansiedad social (ASC) y, sobre los problemas exteriorizados, compuesto por problemas de atención (ATE), hiperactividad-impulsividad (HIP) y conducta desafiante (DES).

Los modelos AFC y SEM se realizaron a través de matrices de correlación policóricas (Barendse, Oort y Timmerman, 2015) y el método de estimación mínimos cuadrados 
ponderados robusto (WLSMV), el cual es robusto con variables discretas no normales (Asparouhov, 2007). El ajuste general de los modelos se evaluó siguiendo las recomendaciones del punto de corte propuesto por Schreiber (2017) (CFI y TLI >. 90 es aceptable $y>.95$ es adecuado; RMSEA $<.08$ es aceptable $y<.06$ es adecuado).

Finalmente, para evaluar las diferencias de salud mental y problemas de regulación entre hombres y mujeres, se utilizó una prueba T para muestras independientes. Adicionalmente, para estimar el tamaño del efecto de dichas diferencias, se utilizó el coeficiente d propuesto por Cohen (1988).

Los análisis estadísticos fueron realizados en los programas estadísticos Statistical Package for the Social Sciences (SPSS), en su versión 25 y Mplus, en su versión 8.2.

\section{Resultados}

Modelos de medida

Según los estándares de ajuste más comunes y recomendados en la literatura (CFl>.95; TLI>.95; RMSEA<.06) (Schreiber, 2017) algunas relaciones entre observaciones se encontraban insuficientemente representadas por los modelos iniciales, con la excepción de la escala de conducta desafiante. Debido a esto, se procedió a depurar iterativamente los modelos de medida a través de la eliminación de ítems redundantes y con cargas factoriales menores a 0.3 (Abada, Olea, Ponsoda y García, 2011), reduciendo de esta forma las escalas. Los detalles del ajuste global de los modelos de medida se presentan en la Tabla 1. 
Alejandra Caqueo-Urízar, Patricio Mena-Chamorro, Jerome Flores-Jara, Marigen Narea-Biscupovich, Matías Irarrázaval-Domínguez

Tabla 1 : Indicadores de ajuste de los modelos de medida iniciales y depurados

\begin{tabular}{cccccccccc}
\hline \multirow{2}{*}{ Model } & Par & $\chi^{2}$ & $d f$ & $p$ & CFI & \multirow{2}{*}{ TLI } & \multirow{2}{*}{ RMSEA } & \multicolumn{2}{c}{ RMSEA Cl $90 \%$} \\
\hline DEP & 70 & 2579.0 & 77 & .000 & .942 & .931 & .118 & .114 & .122 \\
DEP* & 35 & 101.3 & 14 & .000 & .995 & .992 & .052 & .043 & .062 \\
\hline ANS & 50 & 267.4 & 35 & .000 & .987 & .983 & .054 & .048 & .060 \\
ANS* & 40 & 137.0 & 20 & .000 & .992 & .989 & .050 & .042 & .058 \\
\hline ASC & 40 & 2503.4 & 20 & .000 & .600 & .440 & .231 & .224 & .239 \\
ASC $^{*}$ & 20 & 17.5 & 2 & .000 & .992 & .975 & .058 & .035 & .084 \\
\hline ATE & 50 & 1189.7 & 35 & .000 & .865 & .826 & .119 & .113 & .125 \\
ATE* $^{*}$ & 30 & 38.3 & 9 & .000 & .993 & .988 & .038 & .026 & .050 \\
\hline HIP & 50 & 1269.2 & 35 & .000 & .815 & .762 & .123 & .117 & .129 \\
HIP* & 25 & 19.3 & 5 & .000 & .995 & .990 & .035 & .020 & .052 \\
\hline DES & 15 & 0.0 & 0 & .000 & 1.00 & 1.00 & .000 & .000 & .000 \\
\hline REG & 35 & 291.1 & 14 & .000 & .985 & .977 & .092 & .083 & .102 \\
REG* & 25 & 27.0 & 5 & .000 & .997 & .995 & .044 & .028 & .060 \\
\hline
\end{tabular}

Nota: $\mathrm{DEP}=$ Depresión; ANS= Ansiedad; $\mathrm{ASC}=$ Ansiedad social; $\mathrm{ATE}=$ Problemas de atención; HIP= Hiperactividad-impulsividad; $\mathrm{DES}=\mathrm{Conducta}$ desafiante; $R E G=$ Problemas de regulación emocional Par = número de parámetros; $\chi 2=$ chi cuadrado; $d f=$ grados de libertad; $p=$ significado; $\mathrm{CFI}$ = índice de ajuste comparativo; TLI = índice de Tucker-Lewis; RMSEA = Error del cuadrado medio de la raíz de aproximación. $\mathrm{Cl}=$ intervalo de confianza; Low = más bajo; Upp = superior; * Modelo depurado.

Las estimaciones de fiabilidad para los modelos de medida de depresión (DEP) $(\alpha=0.88 ; \omega=$ $0.88)$, ansiedad (ANS) ( $a=0.86 ; \omega=0.86)$, ansiedad social (ASC) $(a=0.74 ; \omega=0.75)$, problemas de atención (ATE) ( $\alpha=0.73 ; \omega=0.74)$, hiperactividad-impulsividad (HIP) $(\alpha=0.70$; $\omega=0.71)$, y problemas de regulación emocional (REG) $(a=0.81 ; \omega=0.82)$ fueron adecuados o al menos suficientes según se utilice alfa de Cronbach (a) u omega jerárquico de McDonald's ( $\omega)$ (Cho y Kim, 2015), salvo la escala de conducta desafiante (DES) $(a=0.69 ; \omega=0.69)$ que está ligeramente por debajo de lo previsto.

Modelo de ecuación estructural (SEM)

Basado en los modelos de medida depurados, se contrastó un modelo de ecuación estructural con los efectos de los problemas de regulación emocional (REG) sobre los problemas interiorizados, compuesto por depresión (DEP), ansiedad (ANS), ansiedad social (ASC) y, sobre los problemas exteriorizados, compuesto por problemas de atención (ATE), hiperactividadimpulsividad (HIP) y conducta desafiante (DES) (Figura 1).

En el modelo se observa que, los problemas de regulación emocional poseen efectos directos 
grandes $(b>.50)$ (Cohen, 1988) sobre todas las variables que componen los problemas interiorizados y exteriorizados, efectos que son distintos de cero en la población. El modelo presentó aceptables niveles de ajuste (CFI= .941; TLI=.936; RMSEA= .053) (Schreiber, 2017), lo cual sugiere que sus relaciones están suficientemente representadas en la población. Finalmente, el modelo es capaz de explicar el $74.6 \%$ de la varianza de depresión, $78.9 \%$ de la varianza de ansiedad, $34.6 \%$ de la varianza de ansiedad social, $43.2 \%$ de la varianza de hiperactividad-impulsividad, $57.3 \%$ de la varianza de problema de atención y $32.4 \%$ de la varianza de conducta desafiante. La representación gráfica del modelo hipotetizado en este estudio se presenta en la Figura 1.

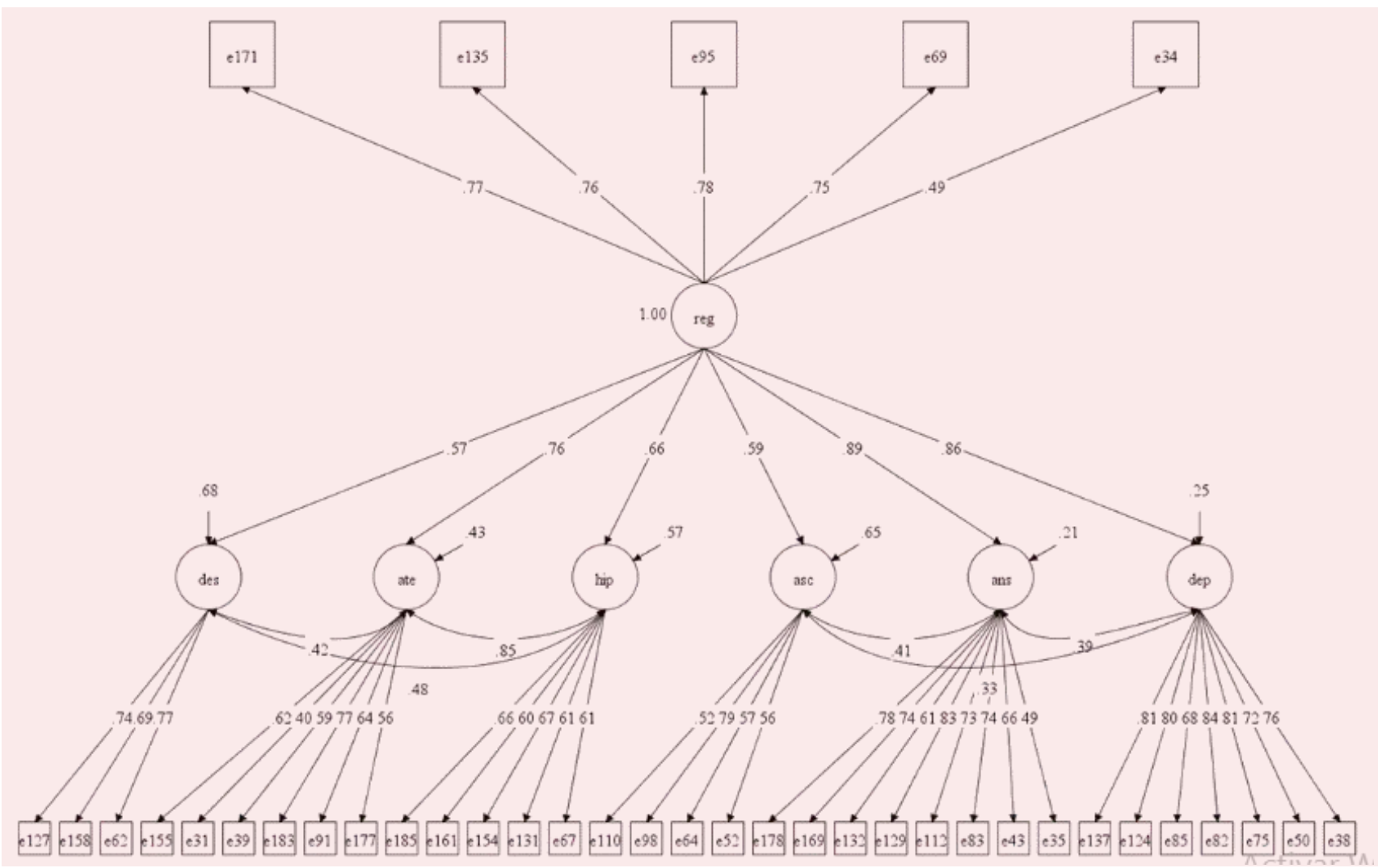

Figura 1 : Modelo de ecuación estructural con los efectos de problemas de regulación emocional sobre problemas interiorizados y exteriorizados

\section{Diferencia entre grupos}

En cuanto a los problemas interiorizados, se observó que las mujeres presentaron mayores síntomas de depresión, ansiedad y ansiedad social que los hombres $(p>0.01)$, diferencias que fueron estadísticamente significativa y presentaron un tamaño del efecto mediano $(d>0.3)$ (Cohen, 1988), mientras que en los problemas exteriorizados no se encontraron diferencias estadísticamente significativas. En cuanto a los problemas de regulación emocional, se 
observó diferencias estadísticamente significativas ( $t=11.71 ; p=0.00)$, con un tamaño del efecto mediano ( $d=0.48$ ) (Cohen, 1988), entre hombres y mujeres, siendo mayor las dificultades en el grupo de mujeres. Los detalles de las diferencias entre estudiantes hombres y mujeres se presentan en la Tabla 2.

Tabla 2 : Comparación entre estudiantes hombres y mujeres en las escalas de vulnerabilidad y problemas exteriorizados e interiorizados

\begin{tabular}{|c|c|c|c|c|c|c|c|c|}
\hline \multirow[b]{2}{*}{ Dimensiones } & \multirow[b]{2}{*}{ Variables } & \multicolumn{2}{|c|}{ Mujer } & \multicolumn{2}{|c|}{ Hombre } & \multirow{2}{*}{$\begin{array}{l}\text { T- } \\
\text { test }\end{array}$} & \multirow[b]{2}{*}{$p$} & \multirow[b]{2}{*}{$d$} \\
\hline & & $M$ & $\mathrm{DE}$ & $M$ & $\mathrm{DE}$ & & & \\
\hline \multirow[t]{3}{*}{ P. interiorizados } & Depresión & 2.2 & 0.9 & 1.9 & 0.7 & 9.38 & $.000^{* *}$ & 0.38 \\
\hline & Ansiedad & 2.7 & 0.9 & 2.3 & 0.7 & 11.17 & $.000^{* *}$ & 0.46 \\
\hline & Ansiedad social & 2.5 & 0.8 & 2.2 & 0.7 & 7.77 & $.000^{* *}$ & 0.32 \\
\hline \multirow{3}{*}{$\begin{array}{l}\text { P. } \\
\text { exteriorizados }\end{array}$} & Problemas de atención & 2.3 & 0.8 & 2.3 & 0.7 & 0.83 & .401 & --- \\
\hline & $\begin{array}{l}\text { Hiperactividad- } \\
\text { impulsividad }\end{array}$ & 2.0 & 0.6 & 2.0 & 0.8 & 2.35 & .059 & - \\
\hline & Conducta desafiante & 1.6 & 0.7 & 1.5 & 0.7 & 0.23 & .811 & --- \\
\hline $\begin{array}{l}\text { Escala de } \\
\text { vulnerabilidad }\end{array}$ & $\begin{array}{l}\text { Problemas de } \\
\text { regulación emocional }\end{array}$ & 2.6 & 1.0 & 2.1 & 0.8 & 11.71 & $.000 * *$ & 0.48 \\
\hline
\end{tabular}

\section{Discusión}

El objetivo de este estudio fue evaluar la asociación entre los problemas de regulación emocional e indicadores de salud mental en adolescentes de la ciudad Arica al Norte de Chile, además de describir y comparar los niveles de problemas interiorizados y exteriorizados entre hombres y mujeres. Este estudio mostró que, los problemas de regulación emocional influyen en la sintomatología depresiva y ansiógena, así como en los comportamientos disruptivos (motricidad excesiva, déficit de control atencional o conductas de desobediencia). Estos resultados son concordantes con los hallazgos encontrados previamente en estudios realizados en países desarrollados (Estados Unidos y Europa) (Garnefski y Kraaij, 2016; Eastabrook et al., 2013; McLaughlin et al., 2011), cuyos resultados también sugieren que la regulación emocional es un factor central en el desarrollo y mantenimiento de trastornos mentales en adolescentes (Aldao, Gee, De Los Reyes y Seager, 2016; Gratz, Weiss y Tull, 2015; Berking, Wirtz, Svald y Hofmann, 2014, Lee et al., 2014; Aldao y Nolen-Hoeksema, 2010; Eisenberg, Spinrad y Eggum, 2010). 
Una razón que podría explicar los hallazgos de este estudio está asociado al carácter dinámico de la regulación emocional, que cambia en función del contexto y del desarrollo de los adolescentes, en este sentido no sólo es un factor de riesgo/protección para la psicopatología, sino también puede ser un resultado en si misma (Aldao et al., 2016). Esto sugiere la necesidad de promover entrenamientos específicos de regulación emocional para adolescentes mediante terapias individuales o grupales. No obstante, ante el difícil acceso a tratamientos en salud mental actualmente en Chile, se podría sugerir que los entrenamientos de regulación emocional se promuevan a través de programas e intervenciones psicoeducativas en los establecimientos educacionales, con la finalidad de generar y articular redes asistenciales de salud mental que permitan llegar a la mayor cantidad de estudiantes.

Aunque los problemas de regulación emocional influyen sobre los problemas interiorizados y exteriorizados, los efectos son mayores cuando se asocia a sintomatología depresiva y ansiosa. Esto sugiere que los adolescentes con dificultades para regular sus emociones son más vulnerables a la aparición de problemas interiorizados que problemas exteriorizados. Esto podría ser explicado por la naturaleza de las variables utilizadas en este estudio, donde los problemas de regulación emocional y problemas interiorizados se expresan y encuentran en el mismo nivel de evaluación (aspectos cognitivos), mientras que los problemas exteriorizados no lo están, ya que son expresados mediante conductas. Esto aumenta la probabilidad de que los efectos de los problemas de regulación sobre problemas exteriorizados se vean disminuido en comparación a los efectos observados sobre los problemas interiorizados (Garnefski, Kraaij y van Etten, 2005).

Los resultados también mostraron la existencia de diferencias en indicadores de salud mental en función del género de los estudiantes, así, las mujeres poseen mayores síntomas depresivos, ansiosos y más problemas de regulación emocional que los hombres. Esta diferencia podría estar siendo explicada por las diferentes formas en que expresan las emociones los hombres y las mujeres, esto asociado a los roles sociales de género, que en parte se regulan por las normas y expectativas sociales de éste, los cuales se encuentran definidas por la comunidad, sobre todo en culturas occidentales, donde se espera que las mujeres sean más expresivas que los hombres (Chaplin, 2015; Fischer y LaFrance, 2015; England, 2010). Sin embargo, este no es el caso para esta muestra, ya que, al parecer, las mujeres estarían presentando dificultad en la expresión de sus emociones. Siendo posible que algunas normas sociales como el enjuiciamiento y valoración constante, características de una cultura patriarcal hegemónica estén reprimiendo esta expresión emocional. 
Evidencia de esto es, el aumento del uso de la rumiación como una estrategia de regulación emocional, que además ha sido identificado como un factor importante en el desarrollo y mantenimiento de sintomatología depresiva en el grupo femenino (Aldao et al., 2016; Zimmermann y lwanski, 2014).

Este estudio posee algunas limitaciones. En primer lugar, se utilizó un instrumento de carácter autoinforme, el cual podría producir sesgos, si es que los sujetos poseen dificultades para comprender y/o identificar sus estados emocionales. Segundo, esta investigación no contiene reportes de padres ni de los profesores, lo cual podría delimitar la comprensión del fenómeno a la capacidad del adolescente para entender sus propias emociones y conductas (De Los Reyes, Alfano, Lau, Augenstein y Borelli, 2016; De Los Reyes et al., 2015). Tercero, el diseño utilizado en investigación, se caracteriza por ser transversal, por lo que no es posible establecer efectos causales entre las variables.

Futuras investigaciones debiesen explorar de manera diferencial los efectos de las estrategias de regulación emocional adaptativas y desadaptativas, así como incorporar otros factores asociados a la regulación emocional disfuncional, como el afrontamiento, autocontrol, apego y resiliencia. Además, con la finalidad de fortalecer los hallazgos de esta investigación, futuros estudios debiesen replicar estos efectos en muestras clínicas de adolescentes, considerando controlar y/o comparar los efectos de las diferentes psicoterapias (terapia de regulación emocional; terapia de aceptación y compromiso; y terapia cognitivo-conductual). En este sentido $y$, considerando los escasos estudios realizados en Latinoamérica, sería interesante explorar estos efectos en otros contextos culturales, como el indígena u/o migrante, ya que la asociación entre regulación emocional y salud mental pudiese estar influenciada por sus creencias y costumbres, así como por la vivencia de estrés por aculturación (Urzúa, CaqueoUrízar y Flores, 2019) que los podría llevar a presentar una dinámica distinta.

\section{Conclusión}

Los resultados obtenidos en esta investigación, muestran que los adolescentes con mayores dificultades para regular sus emociones podrían ser más vulnerables a experimentar problemas interiorizados como exteriorizados, observándose además diferencias entre hombres y mujeres, donde el grupo femenino muestra mayor dificultad en la regulación de las emociones. Estos hallazgos deben ser un insumo para el diseño de futuros programas y/o intervenciones psicoterapéuticas y psicoeducativas en este grupo etario. 


\section{Agradecimientos:}

Esta investigación fue financiada por ANID PIA CIE160007.

\section{Referencias}

Abad, F., Olea, J., Ponsoda, V., y García, C. (2011). Medición en ciencias sociales y de la salud. Madrid: Síntesis.

Ahmed, S., Bittencourt-Hewitt, A., y Sebastian, C. (2015). Neurocognitive bases of emotion regulation development in adolescence. Developmental cognitive neuroscience, 15, 11-25. https://doi.org/10.1016/j.dcn.2015.07.006

Aldao, A., y Nolen-Hoeksema, S. (2010). Specificity of cognitive emotion regulation strategies: A transdiagnostic examination. Behaviour Research and Therapy, 48(10), 974-983. https://doi.org/10.1016/j.brat.2010.06.002

Aldao, A., Gee, D., De Los Reyes, A., y Seager, I. (2016). Emotion regulation as a transdiagnostic factor in the development of internalizing and externalizing psychopathology: Current and future directions. Development and psychopathology, 28(4), 927-946. https://doi.org/10.1017/S0954579416000638

Asparouhov T. (2007). Wald test of mean equality for potential latent class predictors in mixture modeling. Technical appendix. Los Angeles: Muthén y Muthén

Ato, M., López-García, J., y Benavente, A. (2013). Un sistema de clasificación de los diseños de investigación en psicología. Anales de Psicología, 29(3), 1038-1059. https://doi.org/10.6018/analesps.29.3.178511

Bailen, N., Green, L., y Thompson, R. (2019). Understanding emotion in adolescents: A review of emotional frequency, intensity, instability, and clarity. Emotion Review, 17(1), 63-73. https://doi.org/10.1177/1754073918768878

Barendse, M., Oort, F., y Timmerman, M. (2015). Using Exploratory Factor Analysis to Determine the Dimensionality of Discrete Responses. Structural Equation Modeling: A Multidisciplinary Journal, 22(1), 87-101. https://doi.org/10.1080/10705511.2014.934850 
Alejandra Caqueo-Urízar, Patricio Mena-Chamorro, Jerome Flores-Jara, Marigen Narea-Biscupovich, Matías Irarrázaval-Domínguez

Barrett, L., Ochsner, K., Gross, J. (2007). On the automaticity of emotion. In J. Bargh (Ed.), Social psychology and the unconscious: The automaticity of higher mental processes (pp. 173-217). New York, NY: Psychology Press.

Berking, M. (2010). Training emotionaler competenzen (2nd ed.). Berlin: Springer.

Berking, M., Wirtz, C., Svaldi, J., y Hofmann, S. (2014). Emotion regulation predicts symptoms of depression over five years. Behaviour research and therapy, 57, 13-20. https://doi.org/10.1016/j.brat.2014.03.003

Berking, M., Wupperman, P., Reichardt, A., Pejic, T., Dippel, A., y Znoj, H. (2008). Emotionregulation skills as a treatment target in psychotherapy. Behaviour research and therapy, 46(11), 1230-1237. https://doi.org/10.1016/j.brat.2008.08.005

Blakemore, S, y Mills, K. (2014). Is adolescence a sensitive period for sociocultural processing? Annual Review of Psychology, 65, 187-207. https://doi.org/10.1146/annurev-psych-010213115202

Braet, C., Theuwis, L., Van Durme, K., Vandewalle, J., Vandevivere, E., Wante, L., ... Goossens, L. (2014). Emotion Regulation in Children with Emotional Problems. Cognitive Therapy and Research, 38(5), 493-504. https://doi.org/10.1007/s10608-014-9616-x

Chaplin, T. (2015). Gender and emotion expression: A developmental contextual perspective. Emotion Review, 7, 14-21. https://doi.org/10.1177/1754073914544408

Chaplin, T., y Aldao, A. (2013). Gender differences in emotion expression in children: A metaanalytic review. Psychological Bulletin, 139, 735-765. https://doi.org/10.1037/a0030737

Cho, E. y Kim, S. (2015). Cronbach's coefficient alpha: Well-known but poorly understood. Organizational Research Methods, 18(2), 207-230. https://doi.org/10.1177/ 1094428114555994

Cohen, J. (1988). Statistical power analysis for the behavioural sciences. 2nd ed. Hillsdale: Erlbaum.

Cracco, E., Goossens, L., y Braet, C. (2017). Emotion regulation across childhood and adolescence: evidence for a maladaptive shift in adolescence. European Child and Adolescent Psychiatry, 26(8), 909-921. https://doi.org/10.1007/s00787-017-0952-8 
Criss, M., Morris, A., Ponce-Garcia, E., Cui, L., y Silk, J. (2016). Pathways to adaptive emotion regulation among adolescents from low income families. Family Relations, 65(3), 517-529. https://doi.org/10.1111/fare.12202

De Los Reyes, A., Alfano, C., Lau, S., Augenstein, T., y Borelli, J. (2016). Can we use convergence between caregiver reports of adolescent mental health to index severity of adolescent mental health concerns? Journal of Child and Family Studies,25, 109-123. https://doi.org/10.1007/s10826-015-0216-5

De Los Reyes, A., Augenstein, T., Wang, M., Thomas, S., Drabick, D., Burgers, D., y Rabinowitz, J. (2015). The validity of the multi-informant approach to assessing child and adolescent mental health. Psychological bulletin, 141(4), 858. https://doi.org/10.1037/a0038498

de Veld, D., Riksen-Walraven, J., y de Weerth, C. (2012). The relation between emotion regulation strategies and physiological stress responses in middle childhood. Psychoneuroendocrinology, 37,1309-1319. https://doi.org/10.1016/j.psyneuen.2012.01.004

Dvorak, R, Sargent, E, Kilwein, T., Stevenson, B., Kuvaas, N., y Williams, T. (2014). Alcohol use and alcohol- related consequences: Associations with emotion regulation difficulties. The American Journal of Drug and Alcohol Abuse, 40(2), 125- 130. https://doi.org/10.3109/00952990.2013.877920

Eastabrook, J., Flynn, J., y Hollenstein, T. (2013). Internalizing Symptoms in Female Adolescents: Associations with Emotional Awareness and Emotion Regulation. Journal of Child and Family Studies, 23(3), 487-496. https://doi.org/10.1007/s10826-012-9705-y

Ehret, A., Kowalsky, J., Rief, W., Hiller, W., y Berking, M. (2014). Reducing symptoms of major depressive disorder through a systematic training of general emotion regulation skills: protocol of a randomized controlled trial. BMC psychiatry, 14(1), 20. https://doi.org/10.1186/1471-244X-14-20

Eisenberg, N., Spinrad, T., y Eggum, N. (2010). Emotion-related self-regulation and its relation to children's maladjustment. Annual Review of Clinical Psychology, 6, 495-525. https://doi.org/10.1146/annurev.clinpsy.121208.131208

England, P. (2010). The gender revolution: Uneven and stalled. Gender and Society, 24, 149166. https://doi.org/10.1177/0891243210361475 
Errázuriz, P., Valdés, C., Vöhringer, P., y Calvo, E. (2015). Financiamiento de la salud mental en Chile: una deuda pendiente. Revista médica de Chile, 143(9), 1179-1186. https://doi.org/10.4067/S0034-98872015000900011

Fernández-Pinto, I., Santamaría, P., Sánchez-Sánchez, F., Carrasco, M., y del Barrio, V. (2015a) SENA. Sistema de Evaluación de Niños y Adolescentes. Manual técnico. Madrid: TEA Ediciones.

Fernández-Pinto, I., Santamaría, P., Sánchez-Sánchez, F., Carrasco, M., y del Barrio, V. (2015b). SENA. Sistema de Evaluación de Niños y Adolescentes. Manual de aplicación, corrección e interpretación. Madrid: TEA Ediciones.

Fischer, A., y La France, M. (2015). What drives the smile and the tear: Why women are more emotionally expressive than men. Emotion Review, 7, 22-29. https://doi.org/10.1177/ 1754073914544406

Garnefski, N., y Kraaij, V. (2016). Specificity of relations between adolescents' cognitive emotion regulation strategies and symptoms of depression and anxiety. Cognition and Emotion, 32, 1-8. https://doi.org/10.1080/02699931.2016.1232698

Garnefski, N., Kraaij, V., y van Etten, M. (2005). Specificity of relations between adolescents' cognitive emotion regulation strategies and Internalizing and Externalizing psychopathology. Journal of Adolescence, 28(5), 619-631. https://doi.org/10.1016/ j.adolescence.2004.12.009

Gratz, K., Weiss, N., y Tull, M. (2015). Examining emotion regulation as an outcome, mechanism, or target of psychological treatments. Current Opinion in Psychology, 3, 85-90. https://doi.org/10.1016/j.copsyc.2015.02.010

Gross, J., y Thompson, R. (2007). Emotion regulation: Conceptual foundations. In J. Gross (Ed.), Handbook of emotion regulation (pp. 3-24). New York, NY: Guilford Press.

Gross, J., Sheppes, G., y Urry, H. (2011). Cognition and Emotion Lecture at the 2010 SPSP Emotion Preconference. Cognition and Emotion, 25(5), 765-781. https://doi.org/10.1080/ 02699931.2011 .555753 
Hervás, G. y Vázquez, C. (2006). La regulación afectiva: modelos, investigación e implicaciones para la salud mental y física. Revista de Psicología General y Aplicada, 59(1-2), 9-36. https://tinyurl.com/y66s4gwk

Lee, B., Heimer, H., Giedd, N., Lein, E., Šestan, N., Weinberger, D., y Casey, B. (2014). Adolescent mental health-opportunity and obligation. Science, 346(6209), 547-549. https://doi.org/10.1126/science. 1260497

Lee, E., y Bukowski, W. (2012). Co-development of internalizing and externalizing problem behaviors: Causal direction and common vulnerability. Journal of adolescence, 35(3), 713729. https://doi.org/10.1016/j.adolescence.2011.10.008

Lenz, A., Del Conte, G., Hollenbaugh, K., y Callendar, K. (2016). Emotional regulation and interpersonal effectiveness as mechanisms of change for treatment outcomes within a DBT program for adolescents. Counseling Outcome Research and Evaluation, 7(2), 73-85. https://doi.org/10.1177/2150137816642439

McLaughlin, K., Hatzenbuehler, M., Mennin, D., y Nolen-Hoeksema, S. (2011). Emotion dysregulation and adolescent psychopathology: A prospective study. Behaviour research and therapy, 49(9), 544-554. https://doi.org/10.1016/j.brat.2011.06.003

Mennin, D., y Fresco, D. (2009). Emotion regulation as an integrative framework for understanding and treating psychopathology. In A. M. Kring, y D. S. Sloan (Eds.), Emotion regulation and psychopathology (pp. 356-379). New York, NY: Guilford Press.

Mennin, D., Fresco, D., O'Toole, M, y Heimberg, R. (2018). A randomized controlled trial of emotion regulation therapy for generalized anxiety disorder with and without co-occurring depression. Journal of consulting and clinical psychology, 86(3), 268. https://doi.org/10.1037/ccp0000289

Nolen-Hoeksema, S. (2012). Emotion regulation and psychopathology: The role of gender. Annual review of clinical psychology, 8, 161-187. https://doi.org/10.1146/annurev-clinpsy032511-143109

Nolen-Hoeksema, S., y Aldao, A. (2011). Gender and age differences in emotion regulation strategies and their relationship to depressive symptoms. Personality and Individual Differences, 51(6), 704-708. https://doi.org/10.1016/j.paid.2011.06.012 
Oram, R., Ryan, J., Rogers, M., y Heath, N. (2017). Emotion regulation and academic perceptions in adolescence. Emotional and Behavioural Difficulties, 22(2), 162-173. https://doi.org/10.1080/13632752.2017.1290896.

Otzen, T., y Manterola, C. (2017). Técnicas de Muestreo sobre una Población a Estudio. International Journal of Morphology, 35(1), 227-232. https://doi.org/10.4067/S071795022017000100037

Polanczyk, G., Salum, G., Sugaya, L., Caye, A., y Rohde, L. (2015). Annual research review: A meta-analysis of the worldwide prevalence of mental disorders in children and adolescents. Journal of Child Psychology and Psychiatry and Allied Disciplines, 56(3), 345365. https://doi.org/10.1111/jcpp.12381

Sánchez-Sánchez, F., Fernández-Pinto, I., Santamaría, P., Carrasco, M., y del Barrio, V. (2016). SENA, Sistema de Evaluación de Niños y Adolescentes: proceso de desarrollo y evidencias de fiabilidad y validez. Revista de Psicología Clínica con Niños y Adolescentes, 3(2), 23 34. https://tinyurl.com/yyh68853

Saraceno, B., van Ommeren, M., Batniji, R., Cohen, A., Gureje, O., Mahoney, J., ... y Underhill, C. (2007). Barriers to improvement of mental health services in low-income and middleincome countries. The Lancet, 370(9593), 1164-1174. https://doi.org/10.1016/S01406736(07)61263-X

Saxena, P., Dubey, A., y Pandey, R. (2011). Role of emotion regulation difficulties in predicting mental health and well-being. SIS Journal of Projective Psychology y Mental Health, 18, 147-155. https://tinyurl.com/y2ehjhdu

Saxena, S., Thornicroft, G., Knapp, M., y Whiteford, H. (2007). Resources for mental health: scarcity, inequity, and inefficiency. The lancet, 370(9590), 878-889. https://doi.org/10.1016/S0140-6736(07)61239-2

Schäfer, J., Naumann, E., Holmes, E., Tuschen-Caffier, B., y Samson, A. (2017). Emotion Regulation Strategies in Depressive and Anxiety Symptoms in Youth: A MetaAnalytic Review. Journal of Youth and Adolescence, 46(2), 261-276. https://doi.org/10.1007/s10964-016-0585-0

Schreiber, J. (2017). Update to core reporting practices in structural equation modeling. Research in Social and Administrative Pharmacy, 13(3), 634-643. https://doi.org/10.1016/ j.sapharm. 2016.06.006. 
Sloan, E., Hall, K., Moulding, R., Bryce, S., Mildred, H., y Staiger, P. (2017). Emotion regulation as a transdiagnostic treatment construct across anxiety, depression, substance, eating and borderline personality disorders: A systematic review. Clinical psychology review, 57, 141-163. https://doi.org/10.1016/j.cpr.2017.09.002

Tamir, M. (2011). The maturing field of emotion regulation. Emotion Review, 3(1), 3-7. https://doi.org/1 0.1177/1754073910388685

Thompson, R. (1994). Emotion regulation: A theme in search of definition. Monographs of the Society for Research in Child Development, 59, 25-52. https://doi.org/10.1111/j.15405834.1994.tb01276.x

Urzúa, A., Caqueo-Urízar, A., y Flores, J. (2019). Fuentes de estrés por aculturación en la infancia y adolescencia. Propuesta del instrumento de medición FEAC-IA. Universitas Psychologica, 18(5), 1-10. https://doi.org/10.11144/Javeriana.upsy18-5.feai

Veilleux, J., Skinner, K., Reese, E., y Shaver, J. (2014). Negative affect intensity influences drinking to cope through facets of emotion dysregulation. Personality and Individual Differences, 59, 96- 101. https://doi.org/10.1016/j.paid.2013.11.012

Zimmermann, P., y lwanski, A. (2014). Emotion regulation from early adolescence to emerging adulthood and middle adulthood: Age differences, gender differences, and emotionspecific developmental variations. International journal of behavioral development, 38(2), 182-194. https://doi.org/10.1177/0165025413515405

\section{Para citar en APA}

Caqueo-Urízar, A., Mena-Chamorro, P., Flores, J., Narea, M., e Irarrázaval, M. (2020). Problemas de regulación emocional y salud mental en adolescentes del norte de Chile. Terapia Psicológica (En línea), 38(2), 203-222. https://doi.org/10.4067/S071848082020000200203 